\title{
Infecções secundárias em pacientes internados por COVID-19: consequências e particularidades associadas
}

\author{
Secondary infections in patients hospitalized for COVID-19: consequences and \\ associated particularities
Infecciones secundarias en pacientes hospitalizados por COVID-19: consecuencias y particularidades asociadas

Tiago Picolo Fernandes ${ }^{1 *}$, Clara Matos de Abreu$^{2}$, Júlio Oliveira Rocha ${ }^{2}$, Larissa de Oliveira Bianchetti ${ }^{3}$, Larissa de Almeida Sales ${ }^{4}$, Marcelo Queiroz Alves ${ }^{5}$, Mariana Eliote Prates ${ }^{2}$, Nicole Miranda Lemes ${ }^{6}$, Suelen Darlane Vieira ${ }^{7}$, Mônica Isaura Corrêa ${ }^{8}$.

\section{RESUMO}

Objetivo: Descrever as principais características e consequências das infecções secundárias em pacientes internados pela COVID-19. Revisão bibliográfica: A infecção por COVID-19 apresenta variabilidade de sinais e sintomas que podem gerar complicações e evoluir para um quadro de Síndrome Respiratória Aguda Grave. A necessidade de internação em Unidades de Terapia Intensiva e ventilação mecânica relacionam-se ao aumento das taxas de coinfecções e superinfecções e piores desfechos clínicos. Os patógenos mais frequentemente associados a essas infecções são Gram positivos, no início da coinfecção, com mudança de perfil, para Gram negativos, ao longo da internação. Observou-se também a coinfecção viral e fúngica, influenciando na gravidade do quadro. O uso de drogas imunossupressoras, com finalidade de frear a atividade inflamatória exacerbada nesses pacientes e a necessidade recorrente de procedimentos invasivos, são fatores de risco bem descritos para o desenvolvimento de infecções. Considerações finais: É fundamental que a vigilância infecciosa rigorosa seja implementada como rotina nos centros de assistência a pacientes com COVID-19, bem como estudos devem ser ampliados no sentido de otimizar o tratamento das infecções secundárias, reduzir seus impactos e, idealmente, preveni-las sempre que possível.

Palavras-chave: Coinfecção, Unidade de terapia intensiva, COVID-19.

\section{ABSTRACT}

Objective: To describe the main characteristics and consequences of secondary infections in patients hospitalized by COVID-19. Literature review: COVID-19 infection presents a variability of signs and symptoms that can lead to complications and progress to Severe Acute Respiratory Syndrome. The need for admission to Intensive Care Units and mechanical ventilation is related to the increase in the rates of coinfections and superinfections and worse clinical outcomes. The pathogens most frequently associated with these infections are Gram positive at the beginning of the coinfection, with a change in profile to Gram negative throughout the hospital stay. Viral and fungal coinfection was also observed, influencing the severity of the condition. The use of immunosuppressive drugs, with the purpose of curbing the exacerbated inflammatory activity in these patients and the recurrent need for invasive procedures, are well-described risk factors for the

\footnotetext{
1 Universidade Nove de Julho (UNINOVE), Bauru - SP. *E-mail: tiagopicolo@uni9.edu.br

2 Universidade Federal dos Vales do Jequitinhonha e Mucuri (UFVJM), Teófilo Otoni - MG.

${ }^{3}$ Faculdade de Medicina de Barbacena (FUNJOBE), Barbacena - MG.

${ }^{4}$ Faculdade de Ciências Médicas e da Saúde de Juiz de Fora (SUPREMA), Juiz de Fora - MG.

${ }^{5}$ Universidade Nove de Julho (UNINOVE), Guarulhos - SP.

${ }^{6}$ Centro Universitário Governador Ozanan Coelho (UNIFAGOC), Ubá - MG.

${ }^{7}$ Universidade Federal de Minas Gerais (UFMG), Belo Horizonte - MG.

${ }^{8}$ Faculdade de Medicina do Vale do Aço (UNIVAÇO), Ipatinga - MG.
} 
development of infections. Final considerations: It is essential that strict infectious surveillance is routinely implemented in care centers for patients with COVID-19, and studies should be expanded in order to optimize the treatment of secondary infections, reduce their impacts and, ideally, prevent them whenever possible.

Key words: Coinfection, Intensive care units, COVID-19.

\section{RESUMEN}

Objetivo: Describir las principales características y consecuencias de las infecciones secundarias en pacientes hospitalizados por COVID-19. Revisión de la literatura: La infección por COVID-19 presenta signos y síntomas variables que pueden conducir a complicaciones y progresar el Síndrome de Dificuldade Respiratória aguda. La necesidad de ingreso en Unidades de Cuidados Intensivos y ventilación mecánica está relacionada con el aumento de las tasas de coinfecciones y sobreinfecciones y peores resultados clínicos. Los patógenos asociados con mayor frecuencia a estas infecciones son los grampositivos al inicio de la coinfección, con cambio de perfil a gramnegativos a lo largo de la estancia hospitalaria. También se observó coinfección vírica y fúngica, lo que influyó en la gravedad de la afección. El uso de fármacos inmunosupresores, con el fin de frenar la actividad inflamatoria exacerbada en estos pacientes y la necesidad recurrente de procedimientos invasivos, son factores de riesgo bien descritos para el desarrollo de infecciones. Consideraciones finales: Es fundamental que en los centros de atención a pacientes con COVID-19 se implemente de forma rutinaria una estricta vigilancia infecciosa, y se deben ampliar los estudios para optimizar el tratamiento de las infecciones secundarias, reducir sus impactos e, idealmente, prevenirlas siempre que sea posible.

Palabras clave: Coinfección, Unidades de cuidados intensivos, COVID-19.

\section{INTRODUÇÃO}

O surgimento da síndrome infecciosa ocasionada pelo coronavírus da Síndrome Respiratória Aguda Grave 2 (SARS-CoV-2) e sua disseminação têm sido um grande desafio mundial. Segundo o Ministério da Saúde, a Coronavirus disease-19 (COVID-19) é uma doença que se caracteriza por um quadro agudo gripal que pode ser associado com febre, tosse, dor de garganta, coriza, seguido ou não de anosmia, ageusia, mialgia, diarreia e cefaleia. Além disso, a doença pode se desenvolver com um quadro mais grave com dispneia, desconforto respiratório e saturação menor que 95\%, caracterizando a Síndrome Respiratória Aguda Grave (SARS) (MINISTÉRIO DA SAÚDE, 2021).

Em pacientes com infecção por COVID-19, observa-se o número expressivo daqueles que necessitam de internação em das Unidades de Terapias Intensivas (UTI), bem como do uso de ventilação mecânica. Associado a esse quadro, verifica-se também o aumento do acometimento desses pacientes por coinfecções ou superinfecções secundárias, visto que é consolidado na literatura que infecções virais predispõem os pacientes a infecções bacterianas, sendo que estas trazem um pior desfecho em relação aos que não as apresentam (FELDMAN C e ANDERSON R, 2021).

As coinfecções ocorrem quando duas ou mais infecções ocorrem concomitantemente. A superinfecção, por sua vez, diz respeito a uma segunda infecção, em seguida à infecção pelo COVID-19. Dados de uma metanálise mostram que 19\% dos indivíduos internados com COVID-19 apresentaram coinfecção, entretanto, a prevalência foi maior em pacientes internados em enfermaria em relação aos da UTI. O estudo demonstrou, ainda, maior prevalência de superinfecções em relação às coinfecções, particularmente em pacientes na terapia intensiva (MUSUUZA JS, et al., 2021).

Estudos que avaliaram os patógenos mais comuns em pacientes coinfectados e super infectados, demonstraram que os mais frequentes foram organismos gram-negativos, como Enterobacteriaceae, com Escherichia coli, e Pseudomonas aeruginosa, além da Staphylococcus aureus. O sítio de infecção mais comum foi o pulmonar, seguido de bacteremia, infecção do trato urinário e pele (NASIR N, et al., 2021). 
Além disso, verificou-se que pacientes infectados por COVID-19 e que apresentaram coinfecções foram admitidos mais frequentemente em UTIs e, nos casos de superinfecções, apresentaram maior tempo de hospitalização e maior mortalidade (VIDAL CG, et al., 2020). Em Wuhan, cidade chinesa onde teve início a pandemia, $50 \%$ dos pacientes que evoluíram para óbito apresentaram infecção secundária e $31 \%$ dos pacientes intubados apresentaram pneumonia associada à ventilação mecânica (ZHOU F, et al., 2020).

Os pacientes internados que apresentaram casos moderados a graves de COVID-19 apresentaram como fatores de risco para infecção secundária o grau de severidade da doença e o uso de corticosteróides. Além disso, estes pacientes apresentaram altos valores de Proteína C Reativa (PCR) e da razão neutrófilo-linfócito (NASIR N, et al., 2021).

Diante desse cenário, torna-se imprescindível o estudo sobre coinfecções e superinfecções em pacientes hospitalizados com COVID-19, visto que essas estão relacionadas com um pior prognóstico e aumento da mortalidade. Assim, o presente artigo teve como objetivo descrever as principais infecções secundárias que acometem pacientes internados por COVID-19, bem como discutir as consequências associadas a estas infecções.

\section{REVISÃO BIBLIOGRÁFICA}

A doença COVID-19 é caracterizada como uma infecção viral altamente transmissível, cujo agente etiológico é o novo coronavírus zoonótico denominado Coronavírus de Síndrome Respiratória Aguda Grave 2 (SARS-CoV-2). Este patógeno pertence à família Coronaviridae e é um vírus de ácido ribonucleico, cujo material genético é configurado por uma molécula de RNA positivo (KHAN M, et al., 2021; LANSBURY L, et al., 2020). A ocorrência de vírus foi referida pela primeira vez em Wuhan, província de Hubei, China, em dezembro de 2019 e desde então, milhares de vidas têm sido findadas pela dispersão do vírus (SHARIFIPOUR E, et al., 2020).

A transmissão do agente patológico ocorre de indivíduo para indivíduo através do ar ou contato pessoal com secreções contaminadas, que são provenientes da tosse, esternutação, escarro e gotículas de saliva. Dentre os sintomas mais comuns, têm-se a febre, tosse, taquipneia e dispneia (LOTF M, et al., 2020). Outras manifestações clínicas como mialgia, fadiga, diarreia, dor precordial, vômitos, náuseas, odinofagia, congestão nasal, expectoração, anosmia, dispepsia, erupção cutânea, descoloração dos dedos das mãos ou dos pés e conjuntivite viral também são relatadas em diversos estudos (CHEN N, et al., 2020)

Diante dos sintomas apresentados, é possível constatar que o COVID-19 apresenta uma evolução com gravidade variável. Alguns fatores parecem estar associados a uma maior probabilidade de desenvolvimento da forma grave da doença, com dispneia e hipoxemia e, consequentemente, para uma rápida progressão para a SARS. Entre estes fatores estão a idade avançada ( $\geq 65$ anos), comorbidades como hipertensão e diabetes, neutrofilia e linfocitopenia (WU C, et al., 2020).

Uma vez que o manejo da SARS pode necessitar de cuidados intensivos, tem-se observado o surgimento de coinfecções e superinfecções nos pacientes que foram admitidos na UTI, sobretudo naqueles com utilização de ventilação mecânica, cateteres, e que apresentaram tempo prolongado de internação hospitalar. É importante ressaltar que, quando uma infecção ocorre concomitantemente à infecção precedente, a mesma é definida como coinfecção. Em contrapartida, a intercorrência de uma infecção após a infecção antecedente é referida como superinfecção, principalmente quando se trata de uma etiologia advinda de microrganismos que se tornaram resistentes após a utilização de antibióticos (FELDMAN C e ANDERSON R, 2021; HUANG C, et al., 2020).

Na literatura, foram observadas diferentes métricas e tipos de estudos para avaliar o grau de contaminação ou infecção por microrganismos secundários ao SARS-CoV-2. Em uma revisão sistemática acerca de infecções secundárias em pacientes hospitalizados com COVID-19, foi demonstrada uma taxa de coinfecções com outros patógenos de $19 \%$, enquanto as taxas de superinfecções foram de $24 \%$, e ambas associadas a maior mortalidade. Entretanto, em pacientes superinfectados, foi observada uma maior prevalência de comorbidades e utilização de ventilação mecânica (MUSUUZA JS, et al., 2021). 
Já de acordo com dados do Sistema Nacional de Saúde Britânico (NHS), à admissão hospitalar, a proporção de pacientes adultos internados com colonização ou infecção por bactérias foi pouco frequente, com taxas de $1,6 \%$ à admissão e 5,5\% dentro das primeiras 48 horas. No entanto, tal proporção aumenta concomitantemente com o tempo de internação, a uma taxa de 27/1000 pessoas internadas por dia (BASKARAN V, et al., 2021). Nos Estados Unidos foi verificada uma taxa de $21 \%$ de detecção de outros patógenos entre pacientes hospitalizados durante a infecção por COVID-19, com maior proporção de microrganismos de origem hospitalar, culminando em maior uso de antibioticoterapia e tempo de internação em UTI (PUZNIAK L, et al., 2021).

Em estudo realizado na Itália que comparou pacientes com e sem COVID-19, internados em UTI, verificou, por meio de culturas coletadas sistematicamente, aumento na taxa de coinfecções dentre os pacientes com coronavírus, com taxa de $38,3 \%$ contra $23,8 \%$ naqueles sem a doença (CULTERA R, et al., 2021). Já uma pesquisa chinesa encontrou taxa de infecções bacterianas de 9,5\% entre pacientes com COVID-19, e os achados sugeriram que estes pacientes apresentaram doença mais severa e com maior atividade inflamatória, constituindo um maior risco de morbimortalidade (HE S, et al., 2021).

Os fatores de risco associados ao desenvolvimento de infecções nosocomiais em pacientes com COVID19 já foram descritos em diversos estudos. Em pacientes críticos é comum o uso de drogas imunossupressoras a fim de barrar a "tempestade de citocinas", padrão inflamatório capaz de lesionar intensamente o epitélio pulmonar e acometer outros tecidos, levando à falência múltipla de órgãos. Entretanto, o ambiente criado pela utilização de tais medicamentos, associados a intensa atividade imunológica, deixa 0 organismo suscetível ao desenvolvimento de coinfecções (CULTRERA R, et al., 2021).

Além disso, pacientes hospitalizados por COVID-19, principalmente no que se refere aos cuidados intensivos, estão sob o risco de infecções associadas aos cuidados em saúde. A admissão em UTI se relaciona a diferentes tipos de procedimentos invasivos como a realização de acessos intravenosos, cânula nasal de alto fluxo, acessos centrais relacionados à hemodiálise e a Ventilação Mecânica Invasiva (VMI). Tais métodos criam comunicação direta entre o organismo e o ambiente externo, aumentando os riscos de desenvolver infecções secundárias (ZHANG H, et al., 2020; RAWSON TM, et al., 2020).

Zhang $\mathrm{H}$, et al. (2020) reforçam a VMI como um dos pilares do tratamento em pacientes críticos infectados pelo Sars-CoV-2. Embora essa estratégia prolongue as chances de sobrevida do paciente, tal procedimento eleva os riscos de desenvolvimento de complicações. Os autores ainda descreveram o aumento de casos de infecções secundárias em pacientes que utilizaram VMI e alertam para a importância da vigilância infecciosa e início de antibioticoterapia o mais célere possível a fim de otimizar o prognóstico.

Ainda quanto aos fatores relacionados às infecções secundárias, Elabbadi A, et al. (2021) compararam os dados de indivíduos com COVID-19 que desenvolveram coinfecção precoce e os que não evoluíram com tal quadro durante a internação hospitalar. Os autores concluíram que a única característica em comum entre aqueles com alguma infecção secundária foi maior propensão à linfopenia, a despeito do que se refere a presença de comorbidades ou outras características do quadro clínico-laboratorial. Ripa M, et al. (2021) acrescentam a associação entre a necessidade de internação em UTI em até 48 horas após admissão hospitalar como risco aumentado para desenvolvimento de infecções secundárias.

Das infecções nosocomiais típicas de pacientes nas UTIs, destacam-se a pneumonia associada à ventilação mecânica, traqueobronquite, infecção do trato urinário e infecção da corrente sanguínea relacionada ao cateter vascular. Tais complicações são tardias, geralmente ocorrendo após mais de uma semana de estadia do paciente na UTI, em média (BARDI T, et al., 2021).

Dentre as bactérias mais identificadas em pacientes com coinfecção, os estudos em geral encontraram resultados semelhantes, com prevalência inicial aumentada de bactérias gram positivas, porém com mudança de perfil para gram negativas, à medida que se aumenta o tempo de internação, de ventilação mecânica e de estadia em UTI. Em pacientes coinfectados, Klebsiella pneumonia, Streptococcus pneumoniae, e Staphylococcus aureus foram os patógenos mais comuns. Já entre pacientes com superinfecção, o germe mais identificado foi Acinetobacter spp, principalmente entre pacientes em ventilação mecânica. Também 
foram identificadas coinfecções virais, principalmente pelos vírus influenza $A$ e $B$, e vírus sincicial respiratório (MUSUUZA JS, et al., 2021).

Em uma pesquisa realizada na Itália, verificou-se aumento no isolamento de fungos, principalmente Candida albicans e C. parapsilosis. As bactérias mais prevalentes foram Acinetobacter baumannii, Enterococcus faecalis, E. faecium, Staphylococcus epidermidis e Stenotrophomonas maltophilia. Assim, esses dados corroboram com 0 aumento de incidência de germes gram negativos em pneumonias nosocomiais e associadas à ventilação mecânica, e ao aumento de bactérias gram positivas decorrentes de infecções de cateter venoso central (CULTRERA R, et al., 2021).

Assim como as coinfecções e superinfecções bacterianas, as coinfecções e superinfecções virais e fúngicas em indivíduos infectados pela COVID-19 podem ocasionar doenças graves com mau prognóstico. A coinfecção bacteriana e fúngica em indivíduos com COVID-19 pode intensificar a inflamação sistêmica do paciente, influenciando no tempo de recuperação e na gravidade dos sintomas apresentados. Isso ocorre devido ao considerável aumento das citocinas pró-inflamatórias, principalmente da IL-6, que se associa a lesão pulmonar grave (CHEN X, et al., 2020). Além disso, o aumento da permeabilidade vascular, a ativação da cascata de coagulação durante a resposta imune e a falha no balanço pró-coagulação e anticoagulação, que predispõem ao desenvolvimento de microtromboses, coagulação intravascular disseminada e falência múltipla de órgãos (JOSE RJ e MANUEL A, 2020).

Com relação à sintomatologia de coinfecções bacterianas clinicamente diagnosticadas, a febre persistente, movimentos respiratórios curtos e difíceis, III sintomas gastrointestinais, intensificação da gravidade do quadro, necessidade de internação na UTI e de suporte ventilatório, uso de glicocorticóides e maior duração da hospitalização são fatores relacionados a esse quadro (SHENGYANG H, et al., 2021).

Nos exames laboratoriais, além da neutrofilia e linfocitopenia, observa-se também o aumento da procalcitonina, da taxa de sedimentação eritrocitária, do D-dímero, da proteína C-reativa, do nitrogênio ureico sanguíneo, da enzima lactato desidrogenase, do peptídeo natriurético cerebral (BNP) e da mioglobina, bem como a diminuição dos níveis de albumina sérica. Essas alterações sugerem a existência de danos a múltiplos órgãos, confirmados pelos achados radiológicos, que apresentam atenuação em vidro fosco à tomografia computadorizada, bem como derrame pleural, em alguns casos (SHENGYANG H, et al., 2021).

A identificação do microrganismo causador, seja bacteriano, viral ou fúngico, é importante para a definição da terapia antimicrobiana e estratégia de manejo em pacientes infectados. Em um estudo realizado na Espanha, verificou-se que pacientes internados na UTI com quadros de superinfecções adquiridas em ambiente hospitalar por Candida albicans apresentaram candidemia, infecção urinária devido ao uso de cateter e complicações por infecção intra-abdominal. Alguns pacientes com COVID-19 que apresentavam doença pulmonar prévia, estavam em ventilação mecânica prolongada e recebiam altas doses de imunossupressores apresentaram traqueobronquite devido à coinfecção por Aspergillus spp (GARCIA-VIDAL C, et al., 2020).

Além disso, é comum que pacientes com COVID-19 apresentem sintomas gastrointestinais, devido à ocorrência de infecção dos enterócitos pelo SARS-CoV-2, causando alterações na microbiota intestinal. Por conseguinte, organismos patogênicos como Streptococcus, Candida spp. e Aspergillus spp. passam a manifestar-se em maior número nesses pacientes e levam a uma desregulação da microbiota intestinal (CHEN X, et al., 2020). Já em casos de coinfecção por cepas de Staphylococcus aureus resistente à meticilina (MRSA), observaram-se complicações graves em pacientes internados na UTI, com significativo aumento da mortalidade, em razão de infecções do trato respiratório inferior. Esses pacientes têm até quatro vezes mais chances de infecção por MRSA quando passam mais de uma semana na enfermaria (SHARIFIPOUR, et al., 2020).

O tratamento com antimicrobiano empírico e estratégias de manejo de pacientes com coinfecções e/ou superinfecções hospitalizados com a COVID-19 variam. A definição do tratamento é estabelecida conforme a característica do paciente, o tipo de internação realizado em enfermaria ou UTI, com coinfecção adquirida na comunidade e/ou superinfecção adquirida no hospital ou se apresenta quadro de sepse (VIDAL CG, et al., 2021; LANGFORD BJ, et al., 2020). 
Ainda que antibióticos não sejam eficazes para o tratamento do COVID-19, esses medicamentos são prescritos na suspeita ou confirmação da infecção. Diretrizes atuais da Organização Mundial de Saúde (OMS) no manejo de indivíduos graves infectados pelo novo coronavírus indicam o tratamento dirigido ao microrganismo com antibióticos, após a coleta de hemocultura, utilizando agentes de amplo espectro, como cefalosporinas e fluoroquinolonas de terceira geração (LANGFORD BJ, et al., 2020; VIDAL CG, et al., 2021; ELABBADI A, et al., 2021). A duração da antibioticoterapia deve ser a mais breve possível, sendo descontinuada após avaliação clínica e dos exames laboratoriais (VIDAL CG, et al., 2021; OMS, 2021).

Assim, se tratando de infecções nosocomiais em pacientes infectados pela Sars-CoV-2, reforça-se a importância da compreensão dos fatores de risco para ocorrência de coinfecções e superinfecções durante a internação hospitalar, bem como o fortalecimento e treinamento da equipe assistencial a fim de minimizar o risco a esses indivíduos (CHENG K, et al., 2020; SANTIS VD, et al., 2021).

\section{CONSIDERAÇÕES FINAIS}

Diante da revisão realizada, foi possível analisar as particularidades de evolução e gravidade da infecção por COVID-19, sobretudo associada às coinfecções e superinfecções com grande potencial de mortalidade. A internação e o uso da ventilação mecânica são importantes para o tratamento dos casos graves, contudo, é fundamental que a vigilância infecciosa rigorosa seja implementada de rotina nos centros de assistência, para que o uso racional de antimicrobianos seja aplicado, melhorando a sobrevida dos pacientes, com o mínimo impacto no perfil de resistência dos microrganismos. Além disso, deve-se destacar a necessidade de reduzir ações invasivas, sempre que possível, e investir em treinamento e aplicação de técnicas de biossegurança.

\section{REFERÊNCIAS}

1. BARDI T, et al. Nosocomial infections associated to COVID-19 in the intensive care unit: clinical characteristics and outcome. European Journal of Clinical Microbiology \& Infectious Diseases, 2021; 40, 495-502.

2. BASKARAN V, et al. Co-infection in critically ill patients with COVID-19: an observational cohort study from England. Journal of Medical Microbiology, 2021; 70:0001350.

3. CHENG K, et al. Analysis of the Risk Factors for Nosocomial Bacterial Infection in Patients with COVID-19 in a Tertiary Hospital. Risk Management and Healthcare Policy, 2020; 13: 2593-2599

4. CHEN N, et al. Epidemiological and clinical characteristics of 99 cases of 2019 novel coronavirus pneumonia in Wuhan, China: a descriptive study. The Lancet, 2020; 395 (10223): 507-513.

5. CHEN X, et al. The microbial coinfection in COVID-19. Applied Microbiology and Biotechnology, 2020; 104: 77777785.

6. CULTRERA R, et al. Co-Infections in Critically III Patients with or without COVID-19: A Comparison of Clinical Microbial Culture Findings. International Journal of Environmental Research and Public Health, 2021;18(8):4358.

7. ELABBADI A, et al. Bacterial coinfection in critically ill COVID-19 patients with severe pneumonia. Springer Nature, 2021; 49: 559-562.

8. FELDMAN C, ANDERSON R. The role of co-infections and secondary infections in patients with COVID-19. Pneumonia, 2021;13(1): 5 .

9. GARCIA-VIDAL C, et al. Incidence of co-infections and superinfections in hospitalized patients with COVID-19: a retrospective cohort study. Clinical Microbiology and Infection, 2021;27(1):83-88.

10. HE S, et al., Clinical characteristics of COVID-19 patients with clinically diagnosed bacterial co-infection: A multi-center study. PLoS ONE, 2021; 16(4): e0249668.

11. HUANG C, et al. Clinical features of patients infected with 2019 novel coronavirus in Wuhan, China. The Lancet, 2020; 395(10223): 497-506.

12. JOSE RJ, MANUEL A. COVID-19 cytokine storm: the interplay between inflammation and coagulation. The Lancet Respiratory Medicine, 2020;8(6):e46-e47.

13. KHAN M, et al. COVID-19: A Global Challenge with Old History. Epidemiology and Progress So Far Molecules, 2021; 26(1):39.

14. LANGFORD BJ, et al. Bacterial co-infection and secondary infection in patients with COVID19: a living rapid review and meta-analysis. Clinical Microbiology and Infection, 2020; 26:1622-1629.

15. LANSBURY L, et al. Co-infections in people with COVID-19: a systematic review and meta-analysis. The Journal of Infection, 2020; 81(2):266-275.

16. LOTFI M, et al. COVID-19: Transmission, prevention, and potential therapeutic opportunities. International Journal of Clinical Chemistry, 2020; 508: 254-266. 
17. MINISTÉRIO DA SAÚDE. Sintomas Coronavírus. 2021. Disponível em <https://www.gov.br/saude/ptbr/coronavirus/sintomas> Acessado em 8 de julho de 2021.

18. MUSUUZA JS, et al. Prevalence and outcomes co-infection and superinfection with SARS-Cov-2 and other pathogens: A systematic review and meta-analysis. PLoS One, 2021;16(5):e0251170.

19. NASIR N, et al. Risk factors for bacterial infections in patients with moderate to severe COVID-19: a case-control study. Journal of Medical Virology, 2021;93(7): 4564-4569.

20. ORGANIZAÇÃO MUNDIAL DA SAÚDE. COVID-19 Clinical management: living guidance. 2021. Disponível em: <https://www.who.int/publications/i/item/WHO-2019-nCoV-clinical-2021-1>. Acessado em: 19 de julho de 2021.

21. RAWSON TM, et al. Bacterial and Fungal Coinfection in Individuals With Coronavirus: A Rapid Review To Support COVID-19 Antimicrobial Prescribing. Clinical Infectious Diseases, 2020;71(9):2459-2468.

22. RIPA M, et al. Secondary infections in patients hospitalized with COVID-19: incidence and predictive factors. Clinical Microbiology and Infection, 2021; 74; 451-457.

23. SANTIS VD, et al. Bacterial infections in critically ill patients with SARS-2-COVID-19 infection: results of a prospective observational multicenter study. Springer Nature, 2021; X:1-10.

24. SHARIFIPOUR E, et al. Evaluation of bacterial co-infections of the respiratory tract in COVID-19 patients admitted to ICU. BMC Infectious Diseases, 2020; 20(1):646.

25. SHENGYANG H, et al. Clinical characteristics of COVID-19 patients with clinically diagnosed bacterial co-infection: $A$ multi-center study. PLoS ONE, 2021; 16(4): e0249668.

26. PUZNIAK L, et al. A multicenter analysis of the clinical microbiology and antimicrobial usage in hospitalized patients in the US with or without COVID-19. BMC Infectious Diseases, 2021; $21: 227$.

27. VIDAL CG, et al. Incidence of co-infections and superinfections in hospitalized patients with COVID-19: a retrospective cohort study. Clinical Microbiology and Infection, 2021; 27: 83-88.

28. WU C, et al. Risk Factors Associated With Acute Respiratory Distress Syndrome and Death in Patients With Coronavirus Disease 2019 Pneumonia in Wuhan, China. JAMA Internal Medicine 2020; 180(7): 934-943.

29. ZHANG H, et al. Risks and features of secondary infections in severe and critical ill COVID-19 patients. Emerging Microbes \& Infections, 2020; 9(1):1958-1964.

30. ZHOU F, et al. Clinical course and risk fator for mortality of adult inpatients with COVID-19 in Wuhan, China: a restrospective cohort study. The Lancet, 2020; 395(10229):1054-1062. 